\title{
FORTALECIMIENTO DE CAPACIDADES SOBRE ESTILOS DE VIDA SALUDABLE Y PREVENCIÓN DE ITS/VIH Y SIDA EN ADOLESCENTES - HUANCAYO 2007*
}

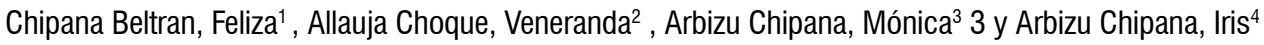 \\ Facultad de Enfermería de la Universidad Nacional del Centro del Perú
}

\begin{abstract}
RESUMEN
Los resultados de la presente investigación nos permite inferir que la aplicación del módulo educativo sobre estilos de vida saludable y prevención de ITS/VIH y SIDA en adolescentes y jóvenes incrementa significativamente el nivel de conocimiento sobre estos temas, A la aplicación del post test en el grupo experimental, incrementan sus conocimientos en un $71,67 \%$ (86), no varían $28,33 \%$ (34); verificado con la pruebas normales donde $\mathrm{z}=$ $-6,40$ y $p=0,00000001$. ). En cuanto al grupo control este incremento sus conocimientos en un $60 \%$ (72), no variaron sus conocimientos $35,67 \%$ (44) y decrementaron $3.33 \%(4 \mid)$, donde $z=-5,35$ y $p=0,00000004$. (ver cuadro $\mathrm{N}^{\circ} 6$ ) Después de la aplicación del Post Test en el nivel de conocimiento global los puntajes dimensionales (sujeto - item), del grupo experimental obtuvieron 38.93\% mientras que el grupo control obtuvieron $29,70 \%$ estos se comprobó con el análisis de perfiles donde el $\mathrm{F}$ calculado es 37.75623 y el $\mathrm{F}$ tabulado con 2/117 grados de libertad es 3.92; donde se acepta la hipótesis alterna en la que se afirma que si existe diferencia significativa entre el grupo control y grupo experimental; y la no existencia de paralelismo de los perfiles de las medias
\end{abstract}

Palabras clave: estilos de vida saludable y prevención de ITS/VIH y SIDA en adolescentes y jóvenes.

\section{CAPACITY BUILDING ON HEALTHY LIFESTYLES AND PREVENTION OF STI / HIV AND AIDS IN ADOLES- CENTS - HUANCAYO 2007}

\begin{abstract}
The results of this investigation allows us to infer that the implementation of educational module on healthy lifestyles and prevention of STI / HIV and AIDS among adolescents and young adults significantly increases the level of knowledge about these issues, the implementation of the post test experimental group increased their knowledge in a $71.67 \%(86)$, do not change $28.33 \%$ (34), verified by standard tests where $z=-6.40 p=0.00000001$. ). Regarding the control group this increased their knowledge by $60 \%(72)$, did not vary their knowledge $35,67 \%$ (44) and decrease 3.33\% (4 |), where $z=-5.35 p=0.00000004$. (see Table 6) After the implementation of the Post Test on the level of global knowledge dimensional scores (subject - item), the experimental group were $38.93 \%$ while the control group were $29.70 \%$ of these were found with the profile analysis where the calculated $\mathrm{F}$ and $F$ is tabulated 37.75623 with 2 / 117 degrees of freedom is 3.92 , which is accepted in an alternate hypothesis which states that if there is significant difference between the control and experimental group and nonexistence of parallelism of the profiles of the means.
\end{abstract}

Key words: styles of healthy life and prevention de its hiv and aids in adolescents and young

\footnotetext{
* Este trabajo de investigación fue recibido el 20/03/2008 retornado para su revisión 26/10/2009 y aprobado para su publicación 16/11/2009

1Email: felizanieves@yahoo.es

2Email: veneach@yahoo.es

${ }^{3}$ Email: monikarbizu@yahoo.es

${ }^{4}$ Email: gabarbizu@hotmail.com
} 


\section{INTRODUCCIÓN}

La pandemia de la infección por VIH es uno de los problemas de salud pública más serios en la historia de la humanidad. Su control involucra desde generación de nuevos conocimientos y desarrollo de nuevas tecnologías, hasta una acción integral y coordinada de los diferentes sectores del Estado y la sociedad civil.

En el Perú esta infección tiene las características de una epidemia concentrada en grupos de alto riesgo, principalmente en varones que tienen sexo con otros varones. Sin embargo es creciente la infección en mujeres y niños. Según la oficina de Epidemiología General del Ministerio de Salud (2003), más de 70.000 personas viven con VIH y SIDA en el país y el principal grupo de edad afectado es el de 25 a 30 años, lo que sugiere que la infección se adquirió durante la adolescencia.

En este contexto, nace la preocupación de establecer estrategias, que permitan, desarrollar capacidades en los adolescentes como, el pensamiento crítico, la toma de decisiones y la solución de problemas a través de una herramienta sencilla elaborada de acuerdo a sus requerimientos. La aplicación del Modulo de "Estilos de Vida Saludable en Prevención de ITS/VIH Y SIDA durante 6 meses de trabajo desarrollando espacios de reflexión individual y colectiva, propiciando el desarrollo de capacidades, cognitivas afectivas, sociales y éticas, que privilegiaron el aprender a aprender y el aprender a convivir en estudiantes del quinto grado de secundaria, consideramos que es un pequeño aporte en la solución de este problema de salud.

Los objetivos planteados fueron: Valorar la práctica de estilos de vida saludable y conocimiento de medidas preventivas sobre ITS, VIH y SIDA antes y después de la aplicación del Módulo Educativo luego, aplicar los talleres educativos del Módulo al grupo de estudio y establecer la efectividad del Módulo Educativo en el cambio de estilos de vida saludable y prevención de ITS. VIH y SIDA. en los estudiantes, la hipótesis que direccionó el estudio fue "el módulo educativo sobre estilos de vida saludable y prevención de ITS, VIH y SIDA aplicado a adolescentes y jóvenes del nivel secundario mejora significativamente el conocimiento sobre medidas preventivas de las ITS,VIH y SIDA a través de la práctica de estilos de vida saludables. "
Para el diseño, ejecución y validación del módulo de soporte educativo se implementó talleres con participación de docentes del centro educativo, y dirección técnica del Ministerio de Salud.

\section{MATERIAL Y METODOS}

El método utilizado es el científico, el analítico sintético y el inductivo deductivo, que nos permitió establecer las características de la población al inicio del estudio y los cambios logrados después de la intervención. una vez determinada la población de estudio y validado el Módulo de Soporte Educativo sobre Estilos de Vida Saludable y Medidas Preventivas sobre ITS, VIH y SIDA, se procedió a aplicar los talleres a los estudiantes donde cada uno de ellos tuvo la oportunidad de aprender sobre estos temas de acuerdo a sus particularidades, luego se realizó las mediciones sucesivas para medir los resultados 0 la efectividad del Módulo .

El tipo de estudio es descriptivo, explicativo y el diseño es experimental, con grupo control. La población de estudio está conformado por 120 estudiantes mayores de 15 años y menores de 18 de género femenino y masculino

\section{RESULTADOS}

Tabla 1. Puntaje dimensional global de conocimientos sobre its en el grupo control y experimental a través del pretest, en los adolescentes del 5 to año de secundaria de las I.E. Santa Isabel, Nuestra Señora de Cocharcas y José Carlos Mariategui.

\begin{tabular}{ccc}
\hline GRUPO & No & PRETEST \\
\hline Control & 120 & 22.06 \\
Experimental & 120 & 21.81 \\
\hline
\end{tabular}

Fuente: Cuestionario - Entrevista

A partir de los puntajes dimensionales sujeto - ítems; notamos la diferencia que existe entre el Grupo control y Experimental antes de recibir el contenido de las dos técnicas educativas; donde, el Grupo Control obtuvo un puntaje dimensional de 22.06 siendo éste mayor que el del Grupo Experimental quien obtuvo un puntaje promedio de 21.81 no encontrándose diferencias significativas- 
Tabla 2. Puntaje dimensional de conocimientos sobre VIH y SIDA en grupos de control y experimental a través del pre y postest, en los adolescentes del 5 to año de secundaria.

\begin{tabular}{cccc}
\hline GRUPO & № & \multicolumn{2}{c}{ TEST } \\
\hline & & PRE & POST \\
Control & 120 & 12.93 & 19.13 \\
Experimental & 120 & 13.13 & 25.01 \\
\hline
\end{tabular}

Fuente: Cuestionario - Entrevista

De los puntos dimensionales sujeto- ítem, se observa que existe diferencia entre los dos grupos cuyos puntajes en el postest son de marcada significancia, en la que el grupo control alcanza 19,13 puntos y el grupo experimental alcanza 25,01 puntos a diferencia de pretest donde lograron 12,93 y 13,13 puntos respectivamente. Si apreciamos en la Figura Nro. 1, notaremos que ambos grupos delinean una pendiente positiva, siendo el grupo experimental el de mayor tendencia positiva. Entonces podemos afirmar que hay incremento significativo de conocimientos porque pasa de un puntaje dimensional de 13,13 en el pre test a 25,01 con el postest con la aplicación de las sesiones educativas. Esto se comprobó a través de las pruebas $F$ donde $F$ calculado es 26,96906 y el $\mathrm{F}$ tabulado con 2/117 grados de libertad es de 3.92 aceptándose de esta manera la hipótesis alterna en la que referimos que sí existe diferencia significativa entre ambos grupos; es decir, aceptamos esta hipótesis de la no existencia del paralelismo de los perfiles de las medias en ambos grupos.

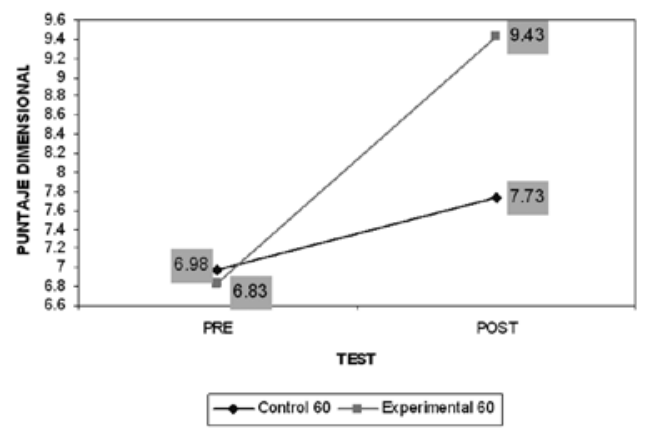

Gráfico 1. Perfil del conocimiento sobre VIH y SIDA de los grupos control y experimental a través del pretest y postest observados en los adolescentes del 5to año de secundaria.
Tabla 3. Puntaje dimensional de conocimientos sobre medidas preventivas de ITS/VIH y SIDA en los grupos de control y experimental a través del pre y postest, en los adolescentes del 5 to año de secundaria.

\begin{tabular}{cccc}
\hline GRUPO & No & \multicolumn{2}{c}{ TEST } \\
\hline & & PRE & POST \\
Control & 120 & 6.98 & 7.73 \\
Experimental & 120 & 9.83 & 9.43 \\
\hline
\end{tabular}

Fuente: Cuestionario - Entrevista

De los puntajes dimensionales sujeto-ítem sobre conocimientos de la medida preventiva de ITS (VIH y SIDA) existe diferencia entre los dos grupos Control y Experimental; donde el grupo experimental pasa de 6,98 puntos en el pre test a 9,43 puntos en el postest, y el grupo control pasa da 6,93 puntos en el pretest a 9,43 puntos en el postest; así podemos afirmar que ambos grupos incrementaron sus conocimientos siendo mayor el de el grupo experimental; esto se observa en la figura $\mathrm{N}^{\circ} 2$ donde las pendientes son más positivas en el grupo experimental que en el de control. Esto se comprobó a través de la prueba $F$ donde el $F$ calculado es 11.43363 y el $F$ tabulado con 2/117 grados de libertad es de 3.92 aceptándose de esta manera la hipótesis alterna en la que referimos que si existe diferencia significativa entre ambos grupos; es decir aceptamos esta hipótesis de la no existencia del paralelismo de los perfiles de las medias en ambos grupo.

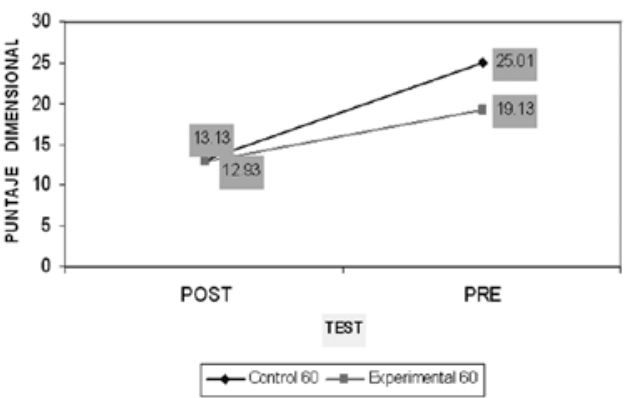

Gráfico 2. Perfil del conocimiento sobre medidas preventivas de ITS/ VIH y SIDA de los grupos control y experimental a través del pre test y post test observados en los adolescentes del 5to año de secundaria - Huancayo 2007 
Tabla 4. Cuadro global de cambios de los conocimientos sobre its/VIH y sida en el grupo control y experimental a observados en los adolescentes del 5to año de secundaria Huancayo - 2007

\begin{tabular}{lcccc}
\hline & \multicolumn{2}{c}{ CONTROL } & \multicolumn{2}{c}{ EXPERIMENTAL } \\
\hline GRUPO & № & $\%$ & № & $\%$ \\
Incrementa & 72 & 60.00 & 86 & 71.67 \\
Invarianza & 44 & 36.67 & 34 & 28.33 \\
Decremento & 2 & 3.33 & 0 & 0 \\
\hline
\end{tabular}

Fuente: pre y postest

En el cuadro se presenta la distinción global de los niveles de incremento de conocimiento sobre ITS/NIH y SIDA donde observamos que incrementaron sus conocimientos en un $71.67 \%$ (86) y no hubo decremento de conocimientos y permanecieron inalterables $28,33 \%$ (17). (Ver figura $\left.\mathrm{N}^{\circ} 3\right)$. En el grupo control encontramos un incremento de $60 \%$ (72) y permanecieron inalterables $36.67 \%$ (44). Esto muy bien nos indica que el grupo experimental ha sido donde hubo mayor ganancia de conocimientos frente al grupo control; esto es respaldado por las pruebas binomiales donde $z=-6,40$ y la probabilidad $(p=$ 0,00000001 ) es mucho más pequeña y significativa.

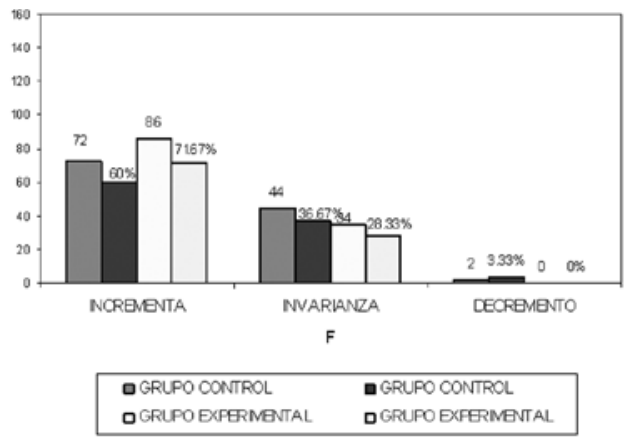

Gráfico 3. Perfil global de la modificación de conocimiento sobre its/vih y sida de los grupos control y experimental observados en los adolescentes del 5to año de secundaria - Huancayo 2007

Tabla 5. Cuadro de modificaciones de conocimientos sobre ITS de los grupos control y experimental en los adolescentes del 5to año de secundaria Huancayo - 2007

\begin{tabular}{lcccc}
\hline & \multicolumn{2}{c}{ CONTROL } & \multicolumn{2}{c}{ EXPERIMENTAL } \\
\hline GRUPO & № & $\%$ & № & $\%$ \\
Incrementa & 78 & 60.00 & 80 & 66.67 \\
Invarianza & 34 & 28.33 & 40 & 33.33 \\
Decremento & 8 & 6.67 & 0 & 0 \\
\hline
\end{tabular}

Fuente: pre y posttest
En este cuadro referido al nivel de conocimiento sobre ITS en el grupo experimental hubo incremento en un $66.67 \%$ (80), no hubo decremento; y permanecen inalterables un 33.33\% (20) (Ver Figura № 4). En el grupo control incrementa sus conocimientos en un $65 \%$ (78). Los conocimientos permanecieron inalterables en un $28.33 \%$ (34)

Esto nos indica claramente que grupo experimental ha sido aquel grupo donde hubo mayor ganancia de conocimientos frente al grupo control, esto es respaldado por las pruebas binomiales aplicados a este grupo se observa que $z=-6,116$ y la probabilidad $(p=0,00000001)$ es mucho mas pequeña y con una diferencia más significativa

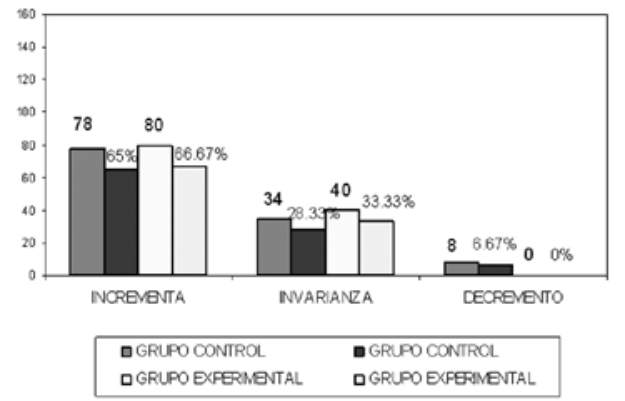

Gráfico 4. Perfil de la modificación de conocimiento sobre its en los grupos control y experimental observados en los adolescentes del 5to año de secundaria - Huancayo 2007

\section{DISCUSIÓN}

La falta de educación sexual y de información sobre las infecciones de transmisión sexual y VIH, así como las dificultades de acceso a programas y medidas de prevención, contribuyen de forma decisiva a la diseminación de estas infecciones entre los jóvenes y es sobre estos factores que se ha trabajado durante el año 2007, buscando disminuir los casos a través de la aplicación de un módulo educativo sencillo donde los adolescente que conformaron el grupo de estudio han tenido la oportunidad de desarrollar capacidades que les permitan adoptar estilos de vida saludables para prevenir estas infecciones.

Uno de los problemas de mayor preocupación en el Perú es la alta tasa de embarazo adolescente, pues 
el $13 \%$ de las adolescentes entre 15 y 19 años ya es madre (11\%) 0 está gestando por primera vez (2\%). El $41.8 \%$ quiso ese hijo/a después ((Encuesta Demográfica y de Salud Familiar 2000-ENDES, 2001).

Entre las causas que determinan el embarazo están el abuso sexual o sexo forzado, uniones tempranas, patrones familiares y culturales y el escaso conocimiento e información en algunas regiones y grupos sociales (Promudeh, Plan Nacional de Igualdad de Oportunidades entre Mujeres y Hombres, 2001).

De las adolescentes, el $14 \%$ ha utilizado alguna vez algún método anticonceptivo, 10\% métodos modernos y el $7.5 \%$ los llamados tradicionales. Sin embargo, solo el $8.8 \%$ utiliza en la actualidad algún método anticonceptivo, $5.6 \%$ uno moderno y $2.9 \%$ uno "tradicional". Aquí observamos con preocupación el abandono por parte de las adolescentes de la utilización de métodos anticonceptivos, sobre todo de métodos modernos, los cuales tienen una protección comprobada (ENDES, 2001).

Otra preocupación es la alta presencia del SIDA entre los jóvenes. El 3.18\% de casos se da en el grupo de 15 a 19 años (hombres 2.9\% y mujeres $0.94 \%$ ). $17.9 \%$ en el grupo entre $20-24$ años (hombres 14\% y mujeres 3.9\%) (Procetss, cifras al 2001). Es importante considerar que debido al largo periodo de evolución de la enfermedad (5 a 10 años), la adquisición de la infección de VIH se produjo durante la etapa adolescente.

Otro dato relevante es que el $12.4 \%$ de las mujeres adolescentes entre 15 y 19 años no sabe del Sida, y el $28 \%$ de ellas no sabe cómo evitarlo. Estas adolescentes se caracterizan por no tener educación $(61.4 \%)$ y el $28.9 \%$ por tener solo educación primaria (ENDES, 2001).

En el Perú, uno de cada 20 adolescentes se ve afectado por una infección de transmisión sexual, con lo cual se convierte en un grupo de alto riesgo para contraer el VIH/Sida. $50.7 \%$ de las mujeres adolescentes entre 15 y 19 años no conoce sobre las infecciones de trasmisión sexual (ITS). ENDES 2001.
En la medida que los adolescentes se conozcan mejor a sí mismos y sean reconocidos por su entorno, podrán valorar sus cualidades y sentirse orgullosos por ello. Asimismo podrán identificar aspectos que deseen cambiar y buscar estrategias adecuadas para lograrlo.

El mundo moderno exige ciudadanos capaces de afrontar problemas, tomar decisiones bajo un proceso razonado, reflexivo y creativo. Es por ello necesario que los estudiantes que cursan la secundaria, desarrollen capacidades para hacer frente a estas exigencias, con prácticas saludables de vida, que permitan su desarrollo óptimo, como seres útiles a la sociedad.

El colegio desempeña un importante papel en la promoción de actitudes responsables y conductas saludables entre los niños y adolescentes. Por ello debe colaborar con la familia en el ámbito de la promoción de valores como el respeto, la igualdad entre sexos y los derechos humanos. La educación sexual contribuye a aplazar la edad de inicio de las relaciones sexuales y, en los adolescentes sexualmente activos, ayuda a disminuir los embarazos no deseados y la infección por VIH y otras enfermedades de transmisión sexual (ETS).

Es necesario tener en cuenta que los programas de prevención fracasarán si sólo tienen en cuenta una forma de prevención como la abstinencia o la fidelidad y no tienen en cuenta del contexto de la vida de los hombres. Fracasarán en las sociedades donde hay poca 0 ninguna oportunidad de hablar de sexo y de la sexualidad ya sea en un contexto público, en la privacidad del hogar. En muchas sociedades, los niños y los adultos no reciben educación sexual. Se argumenta frecuentemente que la educación sexual empuja a los jóvenes a iniciarse en el sexo a una edad temprana. Sin embargo, las investigaciones sobre educación sexual en el mundo confirman todo lo contrario.

La desinformación en materia de salud, es la mejor aliada de la enfermedad. Si de SIDA se trata, no estar al tanto de las vías de transmisión es una de las ignorancias más frecuentes. Desconocer cómo opera el virus y cómo puede dársele batalla, es una de las causas del incremento alarmante de este flagelo de la humanidad. 


\section{CONCLUSIONES}

- El grado de conocimiento acerca de las ITS/VIH y SIDA en adolescentes del 5to año de secundaria de las II.EE. en estudio fue evaluado con el pre test antes de recibir los talleres de módulos educativos; donde obtuvieron un puntaje dimensional de 22,06 para el grupo control y 21,81 puntos para el grupo experimental; esto está verificado con las pruebas de análisis de perfiles

- A la aplicación del postest en el grupo experimental, incrementan sus conocimientos en un $71,67 \%$ (86), no varían $28,33 \%$ (34) y decrementan $0,00 \%$ (0); verificado con la pruebas normales donde $z=-6,40$ y $p=0,00000001$. (ver cuadro $N^{0} 7$ ). En cuanto al grupo control este incremento sus conocimientos en un $60 \%(72)$, no variaron sus conocimientos $35,67 \%$ (44) y decrementaron $3.33 \%(4 \mid)$, donde $z$ $=-5,35$ y $p=0,00000004$. (ver cuadro $\left.N^{\circ} 6\right)$

- Después de la aplicación del Postest en el nivel de conocimiento global los puntajes dimensionales (sujeto - ítem), del grupo experimental obtuvieron $38.93 \%$ mientras que el grupo control obtuvieron 29,70 \% estos se comprobó con el análisis de perfiles donde el $\mathrm{F}$ calculado es 37.75623 y el $\mathrm{F}$ tabulado con 2/117 grados de libertad es 3.92; donde se acepta la hipótesis alterna en la que se afirma que si existe diferencia significativa entre el grupo control y grupo experimental; y la no existencia de paralelismo de los perfiles de las medias.

La técnica de juego educativo es eficaz en el aprendizaje de los adolescentes que permite la participación activa e involucrando al estudiante en su autoaprendizaje.

\section{LITERATURA CITADA}

NetWork en español; Family Health Vol. 17 № 3; Population Reference Bureau (PRB Oficina de Referencias Demográficas) con sede en Washington, 1997; Pág. 9

Díaz Mosto, Jorge. Educación Sexual para Adolescentes; editorial de Libros Técnicos; Lima Perú.

Vásquez Miguel, 1992 Investigación: Los Materiales Didácticos en el Proceso Enseñanza Aprendizaje de Temas de Educación Sexual en los alumnos del 40 grado de secundaria del CE, Mariscal Castilla de el Tambo Huancayo.

Ministerio de Educación ,1996. Guía de Orientación Metodológica de Educación Sexual para 3ro. Y 4to. año de Secundaria; Lima Perú; Pág. 146

Allauja Choque. 2006. Efectividad del programa modular de soporte educativo sobre estilos de vida saludable y prevención de ITS/VIH y SIDA en estudiantes del Colegio secundario Túpac Amaru- Huancayo.

Instituto Nacional de Investigación y Desarrollo de Educación (INIDE). 1995. Centro de Información Educativa (CIE PERU); Métodos de la Nueva Educación (AFA); Editores Importados 3.A; Lima Perú.

Velásquez Carlos Miquel ,1997 Investigación: "Aplicación de Material Didáctico en la Enseñanza y su relación con Rendimiento escolar en los estudiantes del $5^{\circ}$ año de secundaria del CE. Nuestra Señora de Lourdes del Distrito de Pampas del CE. La Victoria de Ayacucho de la ciudad de Huancavelica.

Daniel Vilca Gloria; Santana Javier , 1988. Investigación; Aplicación de medios auxiliares específicos en Ciencias Naturales y su Efectividad en el Aprendizaje en alumnos del Primer Año A Y B del CE. José Carlos Mariátegui.

Ramos Segovia Liliana, 1991. Investigación; Efectividad de Tres Técnicas Educativas en la Enseñanza de Métodos Anticonceptivos a Adolescentes del 50 Año de Secundaria CE. José Ardiles de Pachacamac.

UNISAP. 1995. Modulo de Salud Comunitaria Educación en Salud y la Gestión de Programas Educativos, Unidad 9; Pág. I.

UNíSAP. 1995. Modulo de salud Comunitaria Educación en Salud; Werner y Bower Aprendiendo a Promover la salud; unidad 10; Pág. 1.

Dugas B. Tratado de Enfermería Práctica. Volumen 1. Enfermería y su función Docente .Pág. 49 
Kuethe James 1979. Proceso de Enseñar y Aprender. Principios del Aprendizaje

Bojorques Dolores, Isabel 1933. Didáctica General, Modernos Métodos y Técnicas de Enseñanza Aprendizaje e. Lima Perú. Pág. 17,26
Pizza Claros Joel ,1993. Pedagogía y Tecnología Educativa. Huancayo Perú Pag.2-3.

Beltrán, Carlos, Modulo de Salud Comunitaria Educación en Salud .Unidad 9. Educación en Salud y la Gestión de Programas Educativos; Unidad 9; Pag.4 